This item is the archived peer-reviewed author-version of:

Live demonstration : 3D sonar sensing using low-cost MEMS arrays

\title{
Reference:
}

Kerstens Robin, Steckel Jan.- Live demonstration : 3D sonar sensing using low-cost MEMS arrays

2017 IEEE SENSORS, October 29 - November 1, 2017, Glasgow, Scotland, United Kingdom - ISBN 978-1-5090-1012-7 -

Piscataway, N.J., IEEE, 2017, p. 1

Full text (Publisher's DOI): http://dx.doi.org/doi:10.1109/ICSENS.2017.8234021 


\section{Live Demonstration: 3D sonar sensing using low-cost MEMS arrays}

\author{
Robin Kerstens (robin.kerstens@uantwerpen.be) \\ University of Antwerp \\ FTI CoSys Lab \\ Antwerp, Belgium
}

\author{
Jan Steckel (jan.steckel@uantwerpen.be) \\ University of Antwerp and Flanders Make \\ FTI CoSys Lab \\ Antwerp, Belgium
}

\section{INTRODUCTION}

In recent years, our research group has developed state of the art 3D sonar sensors [1]-[3] which use a low-cost MEMS microphone array for real-time acoustic imaging in air. Using this sensor, various robotic applications have been developed [2], including obstacle avoidance and corridor following and SLAM [1]. The developed sensor is capable of localizing an arbitrary number of reflectors, and generates $2 \mathrm{D}$ (range versus azimuth) or 3D (range versus azimuth and elevation) acoustic images of the environment by emitting a broadband, spatially omnidirectional acoustic emission. This emission is reflected back by the environment to the microphone array. Using array beamforming algorithms an acoustic image of the environment is created, which subsequently can be either visualized or used in various control algorithms. The overview of the developed sensor array can be seen in figure 1 .

\section{Demonstration Setup}

During the demonstration we will showcase several applications of the developed sonar sensor. The first demonstration is real-time 2D acoustic imaging (similar to the image in figure 1 , panel d). The image is updated at $5-10 \mathrm{~Hz}$, and shows the position of the visitors in front of the sensor. The second demonstration is 3D tracking of an object in front of the sonar sensor. A sonar is mounted on a mechanized pan/tilt unit (FLIR PTU46D). The sonar sensor measures the position of a ball in a window around a predefined range (e.g. $1.5 \mathrm{~m}$ ), and tracks the ball in azimuth and elevation. The position of the pan/tilt unit is adapted to track the ball. The final demonstration is a video of robot navigation using only the sonar sensor, similar to the video which accompanies our paper on acoustic flow-based corridor following [2]. Non-functional sonar prototypes will also be available at the demonstration booth to clarify the internal workings of the sensor.

\section{VISITOR EXPERIENCE}

The visitors can interact with the demonstration setup in several ways. The first way of interacting is with the acoustic 2D camera, which shows the position of the users in front of the sonar sensor. The visualization of the acoustic image shows the energy-distribution of the reflections, indicating the position of the users. The second way of interacting with the setup is by manipulating the ball which is to be tracked by the sonar sensor. The ball is mounted on a pole with fishing line, which can be held in front of the sensor and is tracked by the pan/tilt unit.

\section{REFERENCES}

[1] J. Steckel and H. Peremans, "Spatial sampling strategy for a 3D sonar sensor supporting BatSLAM," in 2015 IEEE/RSJ International Conference on Intelligent Robots and Systems (IROS). IEEE, sep 2015, pp. 723-728. [Online]. Available: http://ieeexplore.ieee.org/lpdocs/epic03/wrapper.htm?arnumber=7353452

[2] - "Acoustic Flow-Based Control of a Mobile Platform Using a 3D Sonar Sensor," IEEE Sensors Journal, vol. 17, no. 10, pp. 3131-3141, may 2017. [Online]. Available: http://ieeexplore.ieee.org/document/7888490/

[3] J. Steckel, A. Boen, and H. Peremans, "Broadband 3-D Sonar System Using a Sparse Array for Indoor Navigation," IEEE Transactions on Robotics, vol. 29, no. 1, pp. 161-171, feb 2013. [Online]. Available: http://ieeexplore.ieee.org/lpdocs/epic03/wrapper.htm?arnumber=6331017
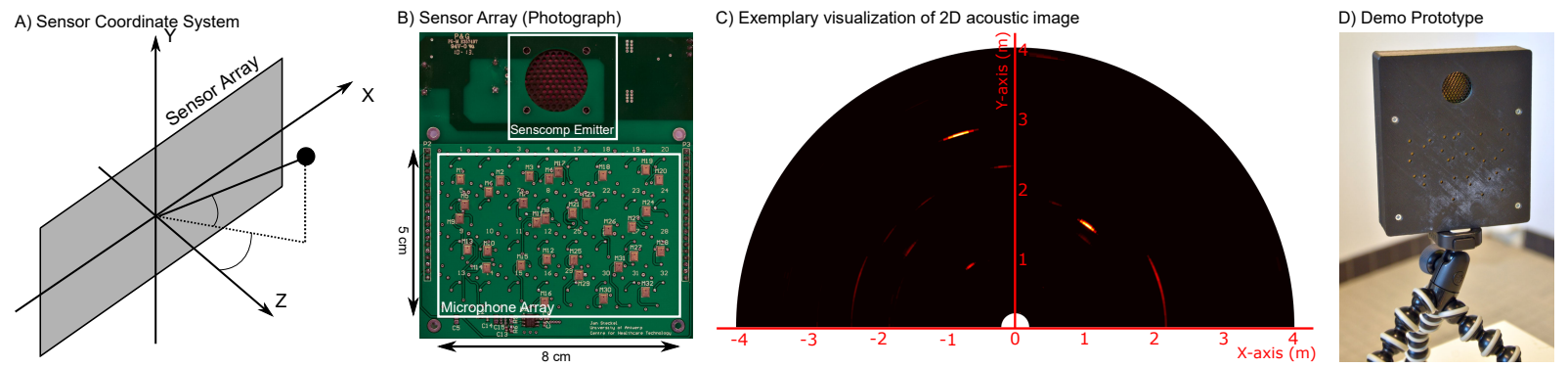

Fig. 1. Overview of the sensor to be showcased on the conference. Panel a) shows the coordinate system attached to the sensor. Panel b) shows the current hardware prototype, with a single emitter and an array of 32 MEMS microphones. Panel c) shows an exemplary 2D acoustic image of an office scene, and panel d) shows the current prototype in it's case, as to be demonstrated on the conference. 